\title{
Effects of Dexamethasone Dose and Timing on Tissue-Engineered Skeletal Muscle Units
}

\author{
Alexie A. Larson ${ }^{a}$ Brian C. Syverud $^{b}$ Shelby E. Florida ${ }^{a}$ Brittany L. Rodriguez ${ }^{b}$ \\ Molly N. Pantelic ${ }^{a}$ Lisa M. Larkin ${ }^{a, b}$ \\ ${ }^{a}$ Molecular and Integrative Physiology, University of Michigan, Ann Arbor, MI, USA; ${ }^{b}$ Biomedical Engineering, \\ University of Michigan, Ann Arbor, MI, USA
}

\section{Keywords}

Tissue engineering $\cdot$ Skeletal muscle $\cdot$ Satellite cells .

Dexamethasone

\begin{abstract}
Our lab showed that administration of dexamethasone (DEX) stimulated myogenesis and resulted in advanced structure in our engineered skeletal muscle units (SMU). While administration of $25 \mathrm{nM}$ DEX resulted in the most advanced structure, $10 \mathrm{~nm}$ dosing resulted in the greatest force production. We hypothesized that administration of $25 \mathrm{nM}$ DEX during the entire fabrication process was toxic to the cells and that administration of DEX at precise time points during myogenesis would result in SMU with a more advanced structure and function. Thus, we fabricated SMU with $25 \mathrm{nM}$ DEX administered at early proliferation (days 0-4), late proliferation (days 3-5), and early differentiation (days 5-7) stages of myogenesis and compared them to SMU treated with 10 nM DEX (days 0-16). Cell proliferation was measured with a BrdU assay (day 4) and myogenesis was examined by immunostaining for MyoD (day 4), myogenin (day 7), and a-actinin (day 11). Following SMU formation, isometric te-
\end{abstract}

(c) 2018 S. Karger AG, Basel

E-Mail karger@karger.com

www.karger.com/cto tanic force production was measured. An analysis of cell proliferation indicated that $25 \mathrm{nM}$ DEX administered at early proliferation (days 0-4) provided $21.5 \%$ greater myogenic proliferation than $10 \mathrm{nM}$ DEX (days 0-4). In addition, $25 \mathrm{~nm}$ DEX administered at early differentiation (days $5-7$ ) showed the highest density of myogenin-positive cells, demonstrating the greatest improvement in differentiation of myoblasts. However, the most advanced sarcomeric structure and the highest force production were exhibited with sustained administration of 10 nM DEX (days $0-16$ ). In conclusion, alteration of the timing of $25 \mathrm{nM}$ DEX administration did not enhance the structure or function of our SMU. SMU were optimally fabricated with sustained administration of $10 \mathrm{nM}$ DEX.

(c) 2018 S. Karger AG, Basel

\section{Introduction}

Volumetric muscle loss, a traumatic loss of skeletal muscle that overwhelms the body's native repair mechanism and results in functional impairment, necessitates surgical intervention [VanDusen et al., 2014]. Limitations to current volumetric muscle loss treatments, in- 


\begin{tabular}{ll}
\hline Abbreviations used in this paper \\
\hline $1 / 2$ RT & half relaxation time \\
DEX & dexamethasone \\
ECM & extracellular matrix \\
ICC & immunocytochemistry \\
MDM & muscle differentiation medium \\
MGM & muscle growth medium \\
SMU & skeletal muscle units \\
TEM & transmission electron microscope \\
TPT & time to peak \\
\hline
\end{tabular}

cluding donor site morbidity and graft tissue availability, present a need for exogenous graft muscle sources [Mertens et al., 2014]. Tissue-engineered skeletal muscle technologies represent a promising graft source for muscle loss repair [Larkin et al., 2006]. However, engineered muscle to date produces forces that are substantially lower than adult muscle, limiting its potential for repair [Williams et al., 2013]. In order for engineered muscle to become a viable means of treatment for volumetric muscle loss, it is essential to advance structural maturity and force production of engineered skeletal muscle towards an adult muscle phenotype. To fabricate phenotypically mature muscle, tissue engineers have used growth factors known to regulate myogenesis in vivo [Allen and Boxhorn, 1989]. Specifically, media is supplemented with growth factors that promote myogenic proliferation, myoblast differentiation, and myotube formation [Husmann et al., 1996]. These studies have shown that providing the appropriate cues to improve each stage of myogenesis is necessary to engineer skeletal muscle with the optimal regenerative and functional potential.

Due to their influence on metabolic processes regulating muscle mass and size, glucocorticoids show promise in providing biochemical cues to enhance the structure and function of engineered skeletal muscle [Morgan et al., 2016]. The effects of dexamethasone (DEX), a synthetic glucocorticoid, have been previously analyzed in vitro with supplementation to myoblast cell lines or muscle cell isolates, as well as in vivo through injection or oral administration; however, the application of DEX in skeletal muscle tissue engineering has yet to be fully explored [Belanto et al., 2010; Inder et al., 2010; Syverud et al., 2016]. At supraphysiological doses, i.e., approximately 10,000 $\mathrm{nM}$ in adult human skeletal muscle and $100 \mathrm{nM}$ in myogenic cell lines, DEX induces skeletal muscle atrophy through increased production of myostatin, a negative regulator of myogenesis, and decreased production of
IGF-1, a hormone that stimulates muscle mass [Inder et al., 2010; Qin et al., 2013]. Specifically, upregulation of myostatin decreases satellite cell proliferation and differentiation, while downregulation of IGF-1 inhibits the PI3-kinase/Akt pathway resulting in stimulation of the FOX03 transcription factor and the downstream ubiquitin ligases MuRF1 and atrogin-1 [Giorgino and Smith, 1995; Kuo et al., 2016]. Eventually, these modifications in myostatin and IGF-1 production stimulate protein degradation, which produces muscle atrophy [Schakman et al., 2008]. Furthermore, researchers have found that addition of pharmacologic doses of DEX to $\mathrm{C} 2 \mathrm{C} 12$ murine myoblast cells can inhibit cell proliferation and protein synthesis processes, which hinder myoblast differentiation and myotube fusion [Belanto et al., 2010]. Interestingly, at similar doses, DEX can augment the production of dysferlin, a calcium-binding transmembrane protein involved in membrane fusion and repair, which promotes myogenic differentiation and enhances myogenic fusion [Desler et al., 1996]. Overall, the diverse effects observed with DEX administration demonstrate the importance of controlling the dose and timing when enhancing in vitro conditions for engineering skeletal muscle.

Previous studies from our lab revealed that sustained administration of $10 \mathrm{nM}$ DEX (days $0-16$ ) yields skeletal muscle units (SMU) with the most advanced form and function to date [Syverud et al., 2016]. Specifically, sustained administration of $10 \mathrm{nM}$ DEX produced SMU with advanced structural development in sarcomeric structure and a 5-fold increase in isometric force production relative to no-DEX controls [Syverud et al., 2016]. Although this study indicated that sustained administration of 25 nM DEX improved myogenic proliferation and differentiation, force production in these SMU was lower and blebbing, an indicator of cell death, was increased compared to SMU without DEX [Syverud et al., 2016]. Therefore, we hypothesized that administration of $25 \mathrm{nM}$ DEX during the entire fabrication process was toxic to the cells and that administration at precise time points during development, rather than sustained administration, would result in the most advanced structure and function. To test our hypothesis, we added $25 \mathrm{nM}$ DEX at early proliferation (days 0-4), late proliferation (days 3-5), and early differentiation (days 5-7) stages, and evaluated myogenic proliferation, differentiation, structural maturation, and force production. We aimed to reproduce the improvements in myogenesis observed with administration of $25 \mathrm{nM}$ DEX early in development and determine whether restricting the administration of $25 \mathrm{nM}$ DEX to one precise stage of myogenesis would produce SMU 
with an improved force production compared to SMU with sustained administration of $10 \mathrm{nM}$ DEX. Overall, we aimed to determine the optimal time to administer the 25 nM DEX dose during our SMU fabrication process, with the goal of improving our engineered skeletal muscle for therapeutic purposes.

\section{Materials and Methods}

\section{Animal Model and Animal Care}

Engineered skeletal muscle was fabricated using soleus muscle isolates and bone marrow from 140- to 160-g female Fischer 344 rats aged 4-6 months obtained from Charles River Laboratories, Inc. (Wilmington, MA, USA). All animals were acclimated to colony conditions for 1 week before any procedure. The animals were fed Purina Rodent Chow 5001 and water ad libitum. All dissection procedures we performed in an aseptic environment, while the animals were in a deep plane of anesthesia induced by intraperitoneal injections of sodium pentobarbital $(65 \mathrm{mg} / \mathrm{kg})$. Supplemental doses of pentobarbital were administered as required to maintain an adequate depth of anesthesia. All animal care and animal surgery procedures were in accordance with the Guide for Care and Use of Laboratory Animals [National Research Council, 1996]; the protocol was approved by the University Committee for the Use and Care of Animals.

\section{Dissection of Muscle and Isolation of Muscle Cells}

Both rat soleus muscles were removed under aseptic conditions and sterilized in 70\% ethanol. Prior to dissociation, the muscles were incubated in $5 \mathrm{~mL}$ of transport medium consisting of Dulbecco's phosphate-buffered saline ( $\mathrm{pH}$ 7.2, catalog No. 14190144; Gibco BRL, Carlsbad, CA, USA) with $2 \%$ antibiotic-antimycotic (catalog No. 15240-062; Gibco BRL) for $5 \mathrm{~min}$. The muscles were then minced using a razor blade, placed under ultraviolet light for 15 min in 15 mL Ham's F12 (catalog No. 11765-047; Gibco BRL), and added to a dissociation solution consisting of $32 \mathrm{U}$ dispase (1.8 U/mg, catalog No. 17105-041; Gibco BRL) and 2390 U type IV collagenase (239 U/mg, catalog No. 17104-019; Gibco $\mathrm{BRL}$ ) in $20 \mathrm{~mL}$ of Ham's F12 nutrient media. The mixture was then kept at $37^{\circ} \mathrm{C}$ with agitation for 90 min to allow the minced muscle to dissociate. Then, the solution was poured through a $100-\mu \mathrm{m}$ filter and centrifuged. The dissociation solution was aspirated off and the cells were resuspended in muscle growth medium (MGM).

\section{SMU Formation and DEX Addition}

SMU were fabricated in individual coated $60-\mathrm{mm}$ polystyrene plates (BD Falcon, Franklin Lakes, NJ, USA), and immunocytochemistry (ICC) was performed on 35-mm plates as described previously [Syverud et al., 2016]. Briefly, a substrate of Sylgard (type 184 silicon elastomer; Dow Chemical Corp., Midland, MI, USA) was cured onto each plate, followed by coating with laminin (Natural Mouse Laminin, catalog No. 23017-015; Gibco BRL) at $1 \mathrm{mg} /$ $\mathrm{cm}^{2}$. Following enzymatic dissociation of satellite cells and fibroblasts from rat soleus muscles, the cell isolation mixture was plated in MGM at a density of 600,000 cells per $60-\mathrm{mm}$ plate and 150,000 cells per $35-\mathrm{mm}$ plate. MGM was composed of $60 \%$ F-12 Kaighn's modification nutrient mixture (catalog No. 21127-022; Gibco BRL), 24\% Dulbecco's modified Eagle's medium (catalog No. 11995-065; Gibco BRL), 15\% fetal bovine serum (catalog No. 10437-028; Gibco BRL), and 1\% ABAM and it was supplemented with $2.4 \mathrm{ng} / \mathrm{mL}$ basic fibroblast growth factor (catalog No. 10018B; Peprotech, Rocky Hill, NJ, USA).

After the initial plating, cells were left to adhere for either 3 or 4 days, depending on the timing of DEX administration. Plates were subsequently fed MGM every 2 days until fully confluent, as indicated by elongated myotube networks across the base of the plate. At this point, the media were switched to muscle differentiation medium (MDM). MDM was composed of 70\% M199 (catalog No. 11150-059; Gibco BRL), 23\% Dulbecco's modified Eagle's medium, $6 \%$ fetal bovine serum, and 1\% ABAM and it was supplemented with $1 \mu \mathrm{L} / \mathrm{mL}$ insulin-transferrin-selenium-X (catalog No. I1884; Sigma-Aldrich, St. Louis, MO, USA) and $14.4 \mu \mathrm{g} / \mathrm{mL}$ ascorbic acid 2-phosphate. After approximately a week on MDM, resupplied every other day, the monolayers were manually delaminated from the plates using a cell scraper, rolled into cylindrical muscle constructs, and held at a length of $3 \mathrm{~cm}$ by engineered bone anchors fabricated from isolated rat bone marrow cells as described previously [VanDusen et al., 2014].

During the SMU fabrication process (described in Fig. 1), 25 nM DEX was added to the media at 3 different time points: early proliferation (days $0-4$ ), late proliferation (days 3-5), and early differentiation (days 5-7). In addition, sustained addition of $10 \mathrm{nM}$ DEX was used as a control, which involved the addition of supplemental doses of $10 \mathrm{nM}$ DEX with each media change. The 3 time points were chosen to optimize the use of $25 \mathrm{nM}$ DEX in enhancement of isolated cell proliferation, differentiation, and maturation of the delaminating monolayer and subsequent 3-D SMU, respectively.

\section{Immunocytochemical Analysis}

During SMU fabrication, a subset of the plates from each experimental group was fixed in $20^{\circ} \mathrm{C}$ methanol for $10 \mathrm{~min}$ and set aside for ICC. Briefly, the plates were washed for $15 \mathrm{~min}$ in $0.1 \%$ Triton X-100 (catalog No. T8787; Sigma) in DPBS (PBST) and blocked with PBST containing 3\% bovine serum albumin (PBST-S, catalog No. A2153; Sigma) for $30 \mathrm{~min}$ at $4^{\circ} \mathrm{C}$. The plates were then incubated overnight at $4^{\circ} \mathrm{C}$ with the primary antibodies diluted in PBST-S. The primary antibodies were used to detect the presence of BrdU (biotin conjugated sheep polyclonal antibody, catalog No. ab2284; Abcam, Cambridge, MA, USA), MyoD (mouse monoclonal antibody, 1:100 dilution, catalog No. 554130; BD Biosciences, San Jose, CA, USA), myogenin (rabbit polyclonal antibody, 1:50 dilution, catalog No. sc-576, Santa Cruz Biotechnology, Dallas, TX, USA), and $\alpha$-actinin (mouse monoclonal antibody, 1:200 dilution, Cat. No. A7752; Sigma). Plates stained with antibodies for BrdU had previously been incubated for $24 \mathrm{~h}$ with a BrdU labeling reagent (1:500 dilution, catalog No. 000103; Life Technologies, Carlsbad, CA, USA) in the culture media. Following 3 washes in PBST, samples were incubated in 1:500 dilutions of Alexa Fluor anti-mouse, anti-rabbit, or streptavidin secondary antibodies (Life Technologies) for $3 \mathrm{~h}$ at room temperature. Following 3 washes in PBST, samples were preserved in Prolong Gold with DAPI (catalog No. P36935; Life Technologies) and coverslipped. The samples were examined and photographed using a Leica Inverted Microscope, and images were analyzed using the Image J software package (NIH, Bethesda, MD, USA). 
Fig. 1. Experimental timeline. To study the effects of DEX on the early and late stages of proliferation and early stage of differentiation, DEX was supplemented from days $0-4$, days $3-5$, and days $5-7$ at a $25 \mathrm{nM}$ dose. Sustained addition of $10 \mathrm{nM}$ DEX, which showed optimal SMU function in our previous study, was used as a control. Throughout the SMU fabrication process, myogenesis was analyzed with immunostaining for proliferation with BrdU and MyoD, differentiation with myogenin, myotube fusion and structural maturation with $a$-actinin, and myotube size and density with light microscopy. Following 3-D SMU formation, isometric tetanic force production was measured to assess functionality.

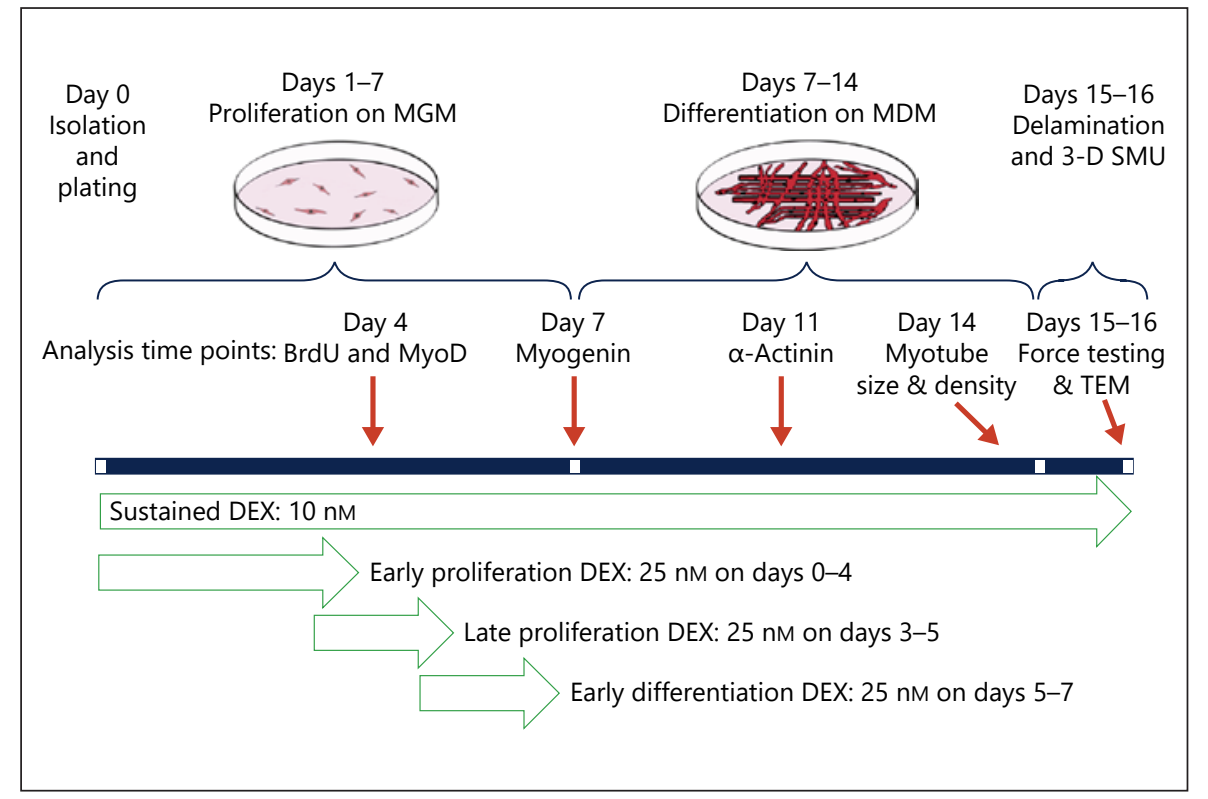

For ICC analysis, plates $(n=6)$ from each experimental group were fixed and stained (on day 4 for BrdU and MyoD, day 7 for myogenin, and day 11 for $\alpha$-actinin). From each plate, 10 areas of $0.6 \mathrm{~mm}^{2}$ were randomly selected and imaged, and the number of positively stained nuclei in each image was counted. Cell proliferation was calculated as a percentage of MyoD-positive nuclei out of BrdU-expressing nuclei. Differentiation of myoblasts into myotubes was determined by measuring the quantity of myogeninpositive cells on each image. To calculate the myotube fusion index from the $\alpha$-actinin images, the number of nuclei associated with an a-actinin-positive myotube containing 4 or more nuclei was divided by the total number of nuclei associated with $\alpha$-actininpositive cells.

\section{Myotube Size and Density}

Fourteen days after the initial plating of isolated cells, light micrographs of the developing monolayers in each experimental group were captured and analyzed. Specifically, 5 areas of $1.4 \mathrm{~mm}^{2}$ from each $60-\mathrm{mm}$ plate $(n=9)$ were randomly selected and imaged. Every myotube from these images was then measured in ImageJ to determine its diameter (in $\mu \mathrm{m}$ ) and the overall density of the myotube network.

\section{SMU Contractile Measurements}

Contractile propertie of SMU ( $n=12$ for $10 \mathrm{nM}$ DEX group and $n=9$ for each $25 \mathrm{nM}$ DEX group) were measured following roll-up into a cylindrical form. The protocol for measuring the contractility of engineered muscle constructs has been described previously [Larkin et al., 1998; Dennis and Kosnik, 2000]. Briefly, the pin on one end of the SMU was raised from the Sylgard and attached to an optical force transducer with a load range of $0-5$ $\mathrm{mN}$ (SI-BAM21-LC KG7A; World Precision Instruments, Sarasota, FL, USA). Platinum wire electrodes were placed along either side of the SMU for electrical field stimulation. The temperature of the construct was maintained at $37^{\circ} \mathrm{C}$ using a heated alumi- num platform. Twitch contractions were elicited using a single $2.5-\mathrm{ms}$ pulse at 60 and $90 \mathrm{~mA}$, whereas the tetanic force was determined using a 1-s train of 2.5-ms pulses at $90 \mathrm{~mA}$ and 60 and $80 \mathrm{~Hz}$. Data files for each peak twitch force and peak tetanic force trace were recorded and subsequently analyzed using LabVIEW 2013 (National Instruments, Austin, TX, USA). The time to peak tension (TPT) was defined as the amount of time from baseline to peak tetanus, and the half relaxation rate $(1 / 2 \mathrm{RT})$ was defined as the amount of time from the onset of relaxation to half of peak tetanus.

\section{Transmission Electron Microscopy Analysis}

Following measures of mechanical function, SMU were fixed overnight at room temperature in $0.1 \mathrm{M}$ phosphate buffer (catalog No. S369-500; Fisher Scientific, Pittsburgh, PA, USA) containing $2.5 \%$ glutaraldehyde (catalog No. 16210; Electron Microscopy Sciences, Hatfield, PA, USA), post-fixed at $4{ }^{\circ} \mathrm{C}$ for $1 \mathrm{~h}$ in $0.1 \mathrm{M}$ phosphate buffer containing 1\% osmium tetroxide (cat No. 19150; Electron Microscopy Sciences), and polymerized in Embed 812 resin (catalog No. 14900; Electron Microscopy Sciences) at $60^{\circ} \mathrm{C}$ for 24 h. Ultra-thin $70-\mathrm{nm}$ sections were then cut and stained with uranyl acetate (catalog No. 22400; Electron Microscopy Sciences) and lead citrate (catalog No. 17900 and 21140; Electron Microscopy Sciences) and imaged using a JEOL JEM-1400 Plus transmission electron microscope (TEM). Images were used to evaluate the structural maturity of SMU by analysis of the development, density, and alignment of collagen fibers and the organization of $\mathrm{Z}$ disks and muscle fibers.

\section{Statistical Analysis}

Values are presented as means \pm SE. Measurements of significant differences between means were performed using GraphPad Prism. Means were compared using either Student's $t$ test when comparing 2 groups or one-way ANOVA tests with Tukey post hoc comparisons. $p<0.05$ was considered statistically significant. 


\section{Results}

Administration of $25 \mathrm{nM}$ DEX Results in the Greatest Increase in Committed Myogenic Progenitors

To determine the effect of DEX dose on the proliferation of isolated myogenic satellite cells, 10 and $25 \mathrm{nM}$ doses of DEX were administered on the day of plating (day 0 ). On day 4, ICC costaining of BrdU, a synthetic thymidine analog, and $\mathrm{MyoD}$, a myogenic transcription factor, was used to identify the total number of proliferating cells and the number of proliferating myogenic cells, respectively [Belanto et al., 2010; Alway et al., 2013]. Analysis of the percentage of cells expressing BrdU and MyoD indicated that plates receiving $25 \mathrm{nM}$ DEX at early proliferation (days $0-4$ ) had $21.5 \%$ more myogenic cell proliferation on average than plates receiving $10 \mathrm{nM}$ DEX at early proliferation $(87.7 \pm 1.7 \%$ vs. $72.2 \pm 2.8 \%$, respectively; Fig. 2). The difference in the number of MyoD-expressing cells between the 2 groups was significant $(p<0.001)$. Therefore, the greatest number of proliferating myogenic nuclei was obtained with administration of $25 \mathrm{nM}$ DEX at early proliferation.

\section{Effects of DEX on Myoblast Differentiation and Myotube Fusion}

The influence of DEX dose and timing on myoblast differentiation into myotubes was evaluated by ICC analysis of myogenin staining on day 7. On average, the administration of $25 \mathrm{nM} \mathrm{DEX}$ at early and late stages of proliferation produced myogenin-positive cell densities of $84.2 \pm 4.0$ and $90.6 \pm 11.9$ cells $/ \mathrm{mm}^{2}$, respectively, which were both significantly lower than the average myogeninpositive cell density of $127.1 \pm 6.4$ cells $/ \mathrm{mm}^{2}$ produced with sustained administration of $10 \mathrm{nM} \mathrm{DEX} \mathrm{(} p=0.012$ and $p=0.036$, respectively; Fig. 3a). In addition, administration of $25 \mathrm{nM}$ DEX at early and late stages of proliferation produced even lower myogenin-positive cell densities compared to the average myogenin-positive cell density of $150.3 \pm 10.3$ cells $/ \mathrm{mm}^{2}$ produced with administration of $25 \mathrm{nM}$ DEX at early differentiation $(p<0.001$ for both stages of proliferation). Furthermore, there was no difference in myogenin expression between sustained administration of $10 \mathrm{nM}$ DEX and administration of 25 nM DEX at early differentiation; however, administration of $25 \mathrm{nM}$ DEX at early differentiation exhibited a $15.4 \%$ greater myogenin-positive cell density compared to sustained administration of $10 \mathrm{nM} \mathrm{DEX}(150.3 \pm 10.3$ vs. $127.1 \pm 6.4$ myogenin-positive cells $/ \mathrm{mm}^{2}$, respectively). Ultimately, myogenin immunostaining showed that differentiation of myoblasts into myotubes was greatest with

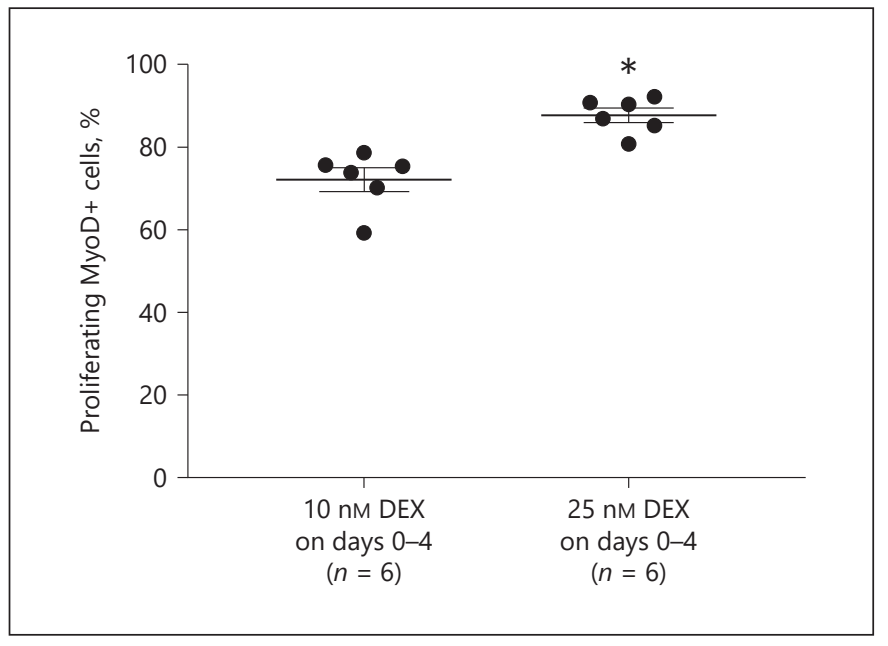

Fig. 2. DEX effects on myogenic proliferation. Percentage of proliferating cells expressing MyoD, indicated by BrdU and MyoD costaining on day 4. Myogenic proliferation was significantly greater with early administration of $25 \mathrm{nM}$ DEX compared to sustained $10 \mathrm{nM}$ DEX $(p<0.001)$. Thick bars show the mean; error bars indicate the SE. * Significant difference from sustained $10 \mathrm{nM}$ DEX.

administration of $25 \mathrm{nM}$ DEX at early differentiation (days 5-7) and sustained administration of $10 \mathrm{nM} \mathrm{DEX}$ (days 0-7).

The role of DEX in terminal differentiation and myotube fusion was examined using staining for $\alpha$-actinin, a structural protein localized at the Z-disc in skeletal muscle cells [Belanto et al., 2010]. Following immunostaining on day 11, the percentage of DAPI-stained positive nuclei associated with $\alpha$-actinin-expressing myotubes containing 4 or more nuclei was assessed to calculate a myotube fusion index value. Plates administered $25 \mathrm{nM}$ DEX at late proliferation exhibited an average fusion index of $79.4 \pm$ $3.0 \%$, which was significantly lower than the average fusion indexes of $91.9 \pm 0.9$ and $91.4 \pm 0.9 \%$ that were obtained with sustained administration of $10 \mathrm{nM}$ DEX and administration of $25 \mathrm{nM}$ DEX at early differentiation, respectively ( $p<0.001$ for both; Fig. 3b). Comprehensively, the most enhanced myoblast differentiation and fusion of myotubes was observed with administration of $25 \mathrm{nM}$ DEX at early differentiation (days 5-7) and sustained administration of $10 \mathrm{nM}$ DEX (days 0-11).

\section{Myotube Growth with DEX Addition}

On day 14 , light microscopy images were captured for analysis of myotube size and density of developing monolayers. Measurements of myotube size showed that the average myotube diameter with administration of 25 


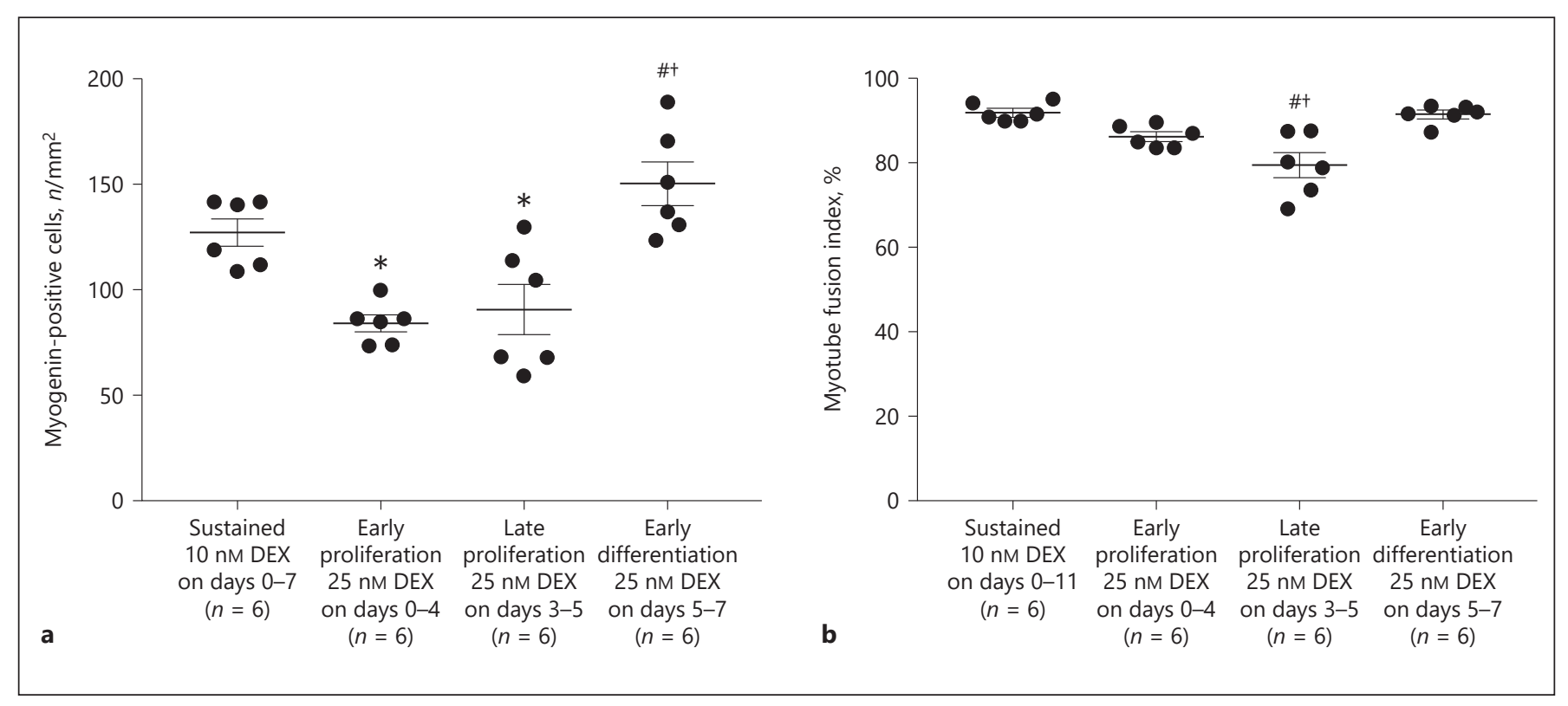

Fig. 3. DEX effects on myoblast differentiation into myotubes. a Myogenin-positive cell density, indicating fusion of myoblasts into myotubes, observed with myogenin immunostaining on day 7. Myogenin-positive cell density was significantly lower with administration of $25 \mathrm{nM}$ at early and late stages of proliferation compared to sustained administration of $10 \mathrm{nM}$ DEX and administration of $25 \mathrm{nM} \mathrm{DEX}$ at early differentiation $(p=0.012, p=0.036$, $p<0.001$, and $p<0.001$, respectively). b Myotube fusion index, representing the percentage of myonuclei associated with a myo- tube containing 4 or more myonuclei, determined from a-actinin and DAPI staining on day 11 . Post hoc analysis indicates a significantly lower myotube fusion index with administration of $25 \mathrm{nM}$ DEX at late proliferation compared to both sustained administration of $10 \mathrm{nM}$ DEX and administration of $25 \mathrm{nM}$ DEX at early differentiation ( $p<0.001$ for both). Thick bars show the mean; error bars indicate SE. * Significant difference from sustained $10 \mathrm{nM}$ DEX. " Significant difference from early proliferation $25 \mathrm{nM}$ DEX. † Significant difference from late proliferation $25 \mathrm{nM}$ DEX.
nM DEX at early and late stages of proliferation was 12.5 \pm 0.7 and $11.9 \pm 0.7 \mu \mathrm{m}$, respectively, which were significantly less than the average myotube diameter of $17.0 \pm$ $1.3 \mu \mathrm{m}$ obtained with sustained administration of $10 \mathrm{nM}$ $\operatorname{DEX}(p=0.015$ and $p=0.005$, respectively; Fig. 4$)$. Furthermore, the average myotube diameter produced with administration of $25 \mathrm{nM}$ DEX at late proliferation was $32.8 \%$ less than the average myotube diameter obtained with administration of $25 \mathrm{nM}$ DEX at early differentiation. The difference in average myotube diameter between administration of $25 \mathrm{nM}$ DEX at late proliferation and administration of $25 \mathrm{nM} \mathrm{DEX}$ at early differentiation was significant $(p=0.045)$. However, there was no difference in average myotube diameter between administration of $25 \mathrm{nM}$ DEX at early differentiation and sustained administration of $10 \mathrm{nM}$ DEX (15.8 \pm 1.1 vs. 17.0 $\pm 1.3 \mu \mathrm{m}$, respectively). Similarly, there were no differences in myotube density between the groups, potentially due to the large intracohort variability. Again, monolayers with the most robust and greatest density of myotubes were observed with administration of $25 \mathrm{nM}$ DEX at early differentiation (days 5-7) and sustained administration of $10 \mathrm{nM}$ DEX (days 0-14).

\section{Sustained Administration of $10 \mathrm{nM}$ DEX Enhances \\ Structural and Contractile Properties of SMU}

To evaluate the structural maturity of the developing monolayers, immunostaining for $\alpha$-actinin on day 11 was evaluated. It was evident that plates with sustained administration of 10 nM DEX exhibited an advanced sarcomeric structure within muscle fibers that were aligned along the length of the SMU (Fig. 5a). TEM images of 3-D SMU with sustained administration of $10 \mathrm{nMDEX}$ showed large myofibers and an abundance of collagen extracellular matrix (ECM) between myofibers (black asterisks in Fig. $5 c$, e). In addition, sarcomeres in longitudinal and transverse orientation were found in TEM images of SMU with sustained administration of $10 \mathrm{nM}$ DEX (diamonds in Fig. 5e). In contrast to plates with sustained administration of $10 \mathrm{nM} \mathrm{DEX}$, an advanced sarcomeric structure was not observed in plates with administration of $25 \mathrm{nM}$ DEX and TEM images of SMU with administra- 

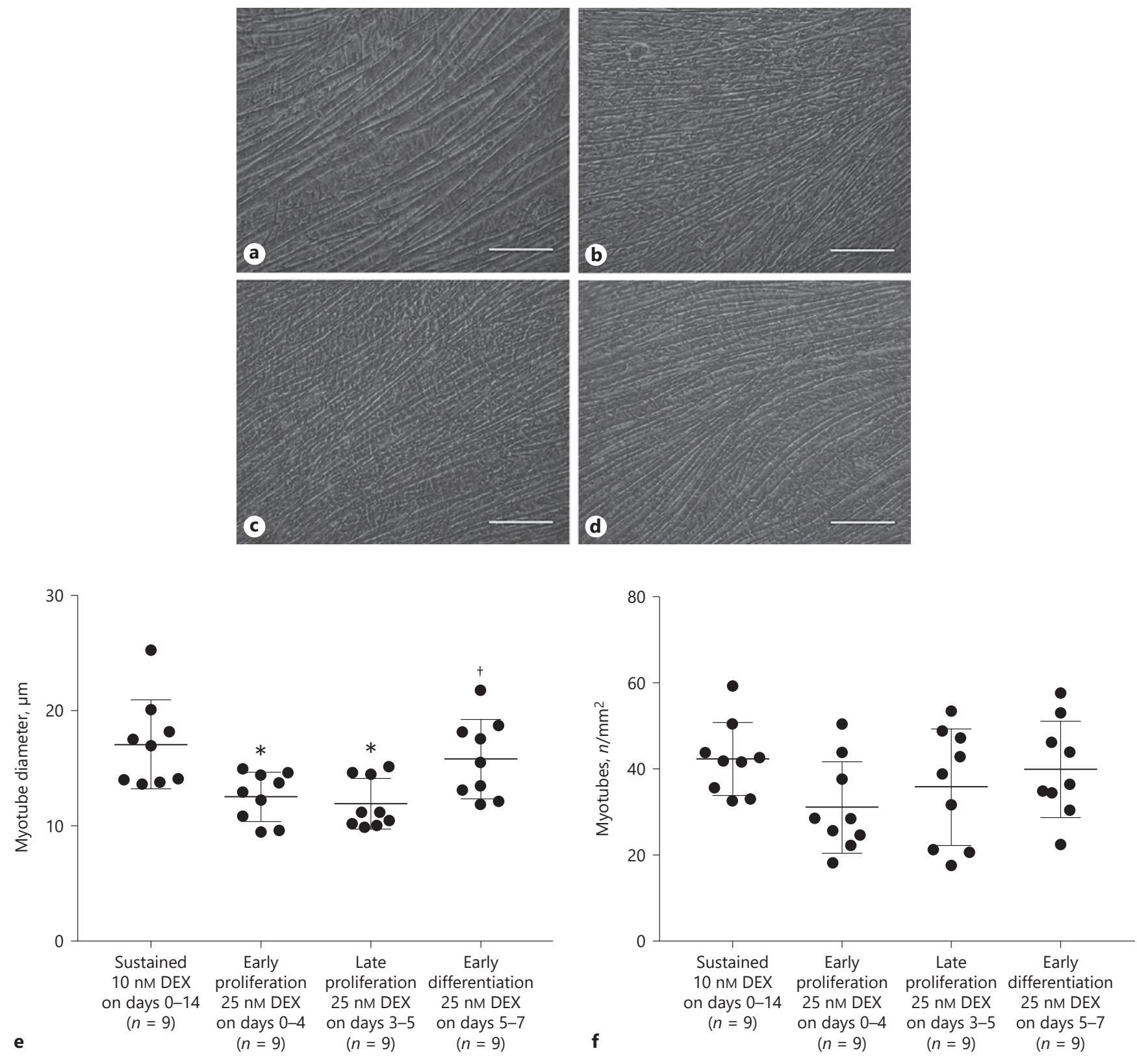

Fig. 4. DEX effects on myotube size and density. Light microscopy images of monolayers were observed before monolayer delamination on day 14. Representative images are shown above for sustained administration of $10 \mathrm{nM}$ DEX (days 0-14) (a), administration of $25 \mathrm{nM} \mathrm{DEX}$ at early proliferation (days $0-4)(\mathbf{b})$, late proliferation (days 3-5) (c), and early differentiation (days 5-7) (d). All images were analyzed for myotube diameter (e) and myotube density (f). ImageJ analysis indicates that the average myotube diameter is significantly lower with administration of early- and lateproliferation $25 \mathrm{nM}$ DEX compared to sustained $10 \mathrm{nM} \operatorname{DEX}(p=$

0.015 and $p=0.005$, respectively). Comparison of $25 \mathrm{nM}$ DEX-receiving groups indicates that the average myotube diameter produced through the administration of $25 \mathrm{nM}$ DEX at late proliferation was significantly lower than that produced with administration of $25 \mathrm{nM}$ DEX at early differentiation $(p=0.045)$. There were no significant differences in myotube density among the 4 groups. Scale bars, $100 \mu \mathrm{m}$. Thick bars show the mean; error bars indicate the SE. * Significant difference from sustained $10 \mathrm{nM}$ DEX. ${ }^{\dagger}$ Significant difference from late proliferation $25 \mathrm{nM}$ DEX. 

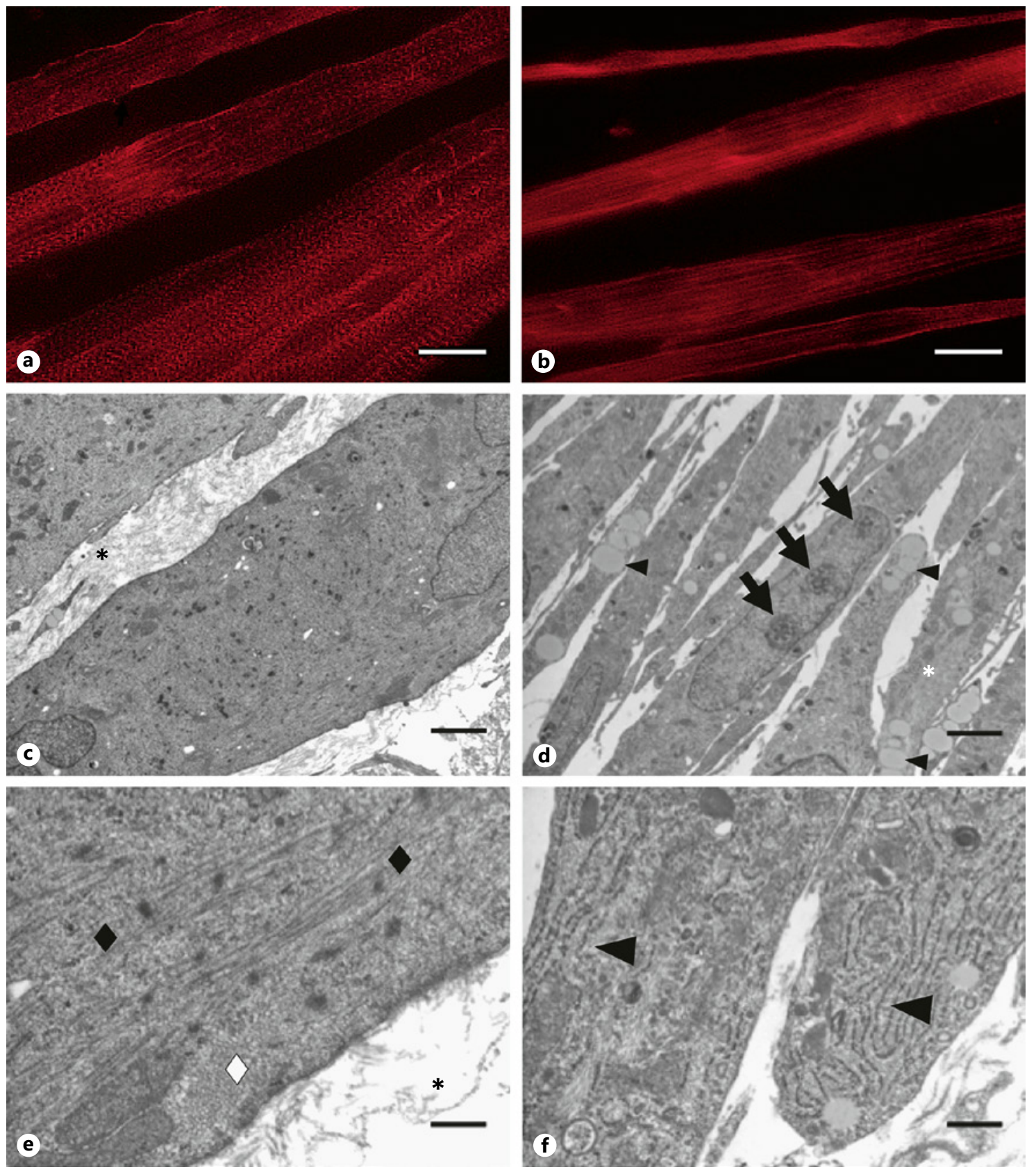

Fig. 5. DEX effects on SMU structural maturation. Representative images of $\alpha$-actinin immunostaining on day 11 following sustained administration of $10 \mathrm{nM} \mathrm{DEX} \mathrm{(days} \mathrm{0-11)} \mathrm{(a)} \mathrm{and} \mathrm{administration}$ of $25 \mathrm{nM}$ DEX at early differentiation (days 5-7) (b). The greatest advancement in sarcomeric structure and alignment of muscle fibers was observed with sustained administration of $10 \mathrm{nM} \mathrm{DEX}$. Transmission electron micrographs of longitudinal sections of SMU following sustained administration of $10 \mathrm{nM}$ DEX (days $0-16)$ (c, e) and administration of $25 \mathrm{nM}$ DEX at early differentiation (days 5-7) (d, f). Images of sustained administration of $10 \mathrm{nM}$ DEX show larger myofibers with an abundance of collagen ECM between myofibers, indicated by theasterisks in (c and e, while images of administration of $25 \mathrm{nM}$ DEX at early differentiation show smaller myofibers and the absence of collagen ECM between myofibers. Black and white diamonds in e indicate sarcomeres in longitudinal and cross orientation along the length of the myofibers observed with sustained administration of $10 \mathrm{nM}$ DEX, respectively. No sarcomeres were observed with administration of $25 \mathrm{nM}$ DEX at early differentiation, but TEM images showed an abundance of myofilaments, lipids droplets, and nucleoli in myonuclei, indicated by the asterisk, arrowheads, and arrows in $\mathbf{d}$, respectively. In addition, the arrowheads in $\mathbf{f}$ indicate an increase in rough endoplasmic reticulum in the cytoplasm of myocytes with administration of $25 \mathrm{nM}$ DEX at early differentiation. Scale bars, $20 \mu \mathrm{m}$ (a, b), $2 \mu \mathrm{m}(\mathbf{c}, \mathbf{d})$, and $400 \mathrm{~nm}(\mathbf{e}, \mathbf{f})$. 


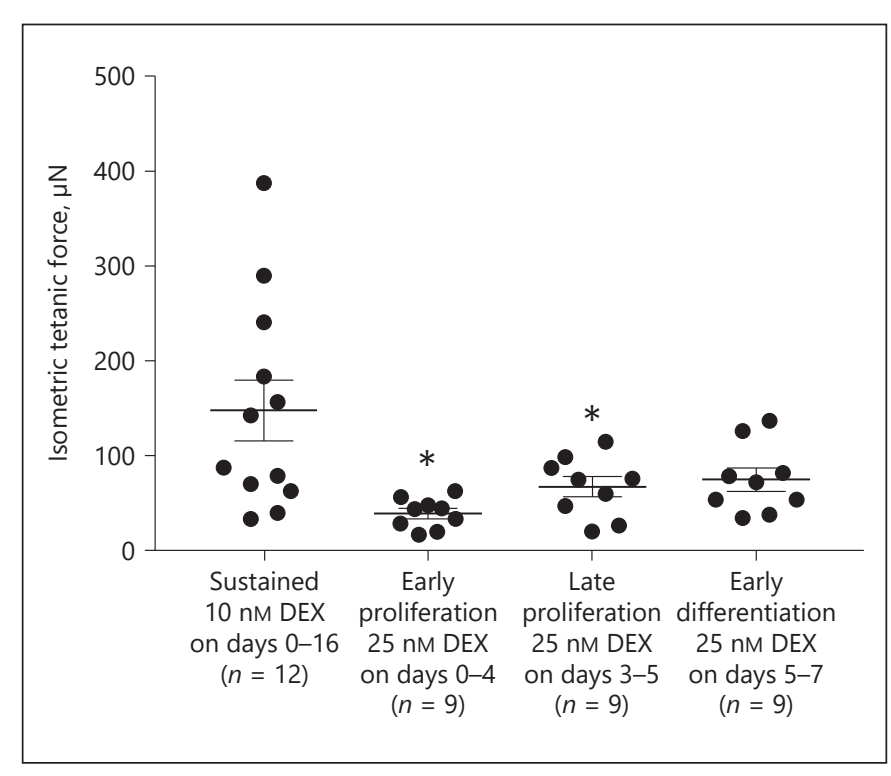

Fig. 6. DEX effects on force production. Functional properties of $3-\mathrm{D}$ SMU assessed on days $15-16$ by measuring the isometric tetanic force production following electrical stimulation. SMU with administration of $25 \mathrm{nM}$ DEX at early and late stages of proliferation exhibited significantly lower average maximum forces than SMU receiving sustained administration of $10 \mathrm{nM} \mathrm{DEX}(p=0.004$ and $p=0.045$, respectively). There were no significant differences in average maximum isometric forces within the $25 \mathrm{nM}$ DEX-receiving cohorts. Thick bars show the mean, and error bars indicate the SE. * Significant difference from sustained $10 \mathrm{nM}$ DEX.

tion of $25 \mathrm{nM}$ DEX at early differentiation showed small myofibers and an absence of collagen ECM between myofibers (Fig. 5b, d). While no sarcomeres were observed in TEM images of SMU with administration of $25 \mathrm{nM} \mathrm{DEX}$ at early differentiation, there was an abundance of unorganized myofilaments and sparsely organized myofilaments in both longitudinal and transverse orientations along the length of the myofibers (asterisk in Fig. 5d). Furthermore, TEM images of SMU with administration of $25 \mathrm{nM}$ DEX at early differentiation showed an increase in intracellular lipid droplets, rough endoplasmic reticulum, and nucleoli in myonuclei (small arrowheads, arrows, and large arrowheads in Fig. 5d, f). Overall, the greatest advancement in structure was obtained with sustained administration of $10 \mathrm{nM}$ DEX.

Following delamination of 3-D SMU, contractile properties were measured in response to electrical stimulation. SMU with administration of $25 \mathrm{nM} \mathrm{DEX}$ at early and late stages of proliferation produced average tetanic forces of $39.4 \pm 5.2$ and $67.3 \pm 10.6 \mu \mathrm{N}$, indicating a 73.3 and $54.4 \%$ lower force than the average tetanic force of $147.7 \pm 31.8 \mu \mathrm{N}$ produced by SMU with sustained admin-

Effects of DEX on Tissue-Engineered SMU istration of $10 \mathrm{nM}$ DEX, respectively (Fig. 6). The differences in average tetanic force between administration of $25 \mathrm{nM}$ DEX at early and late stages of proliferation and sustained administration of $10 \mathrm{nM}$ DEX were significant ( $p=0.004$ and $p=0.045$, respectively). Furthermore, SMU with administration of $25 \mathrm{nM}$ DEX at early differentiation generated an average tetanic force of $74.9 \pm 12.0$ $\mu \mathrm{N}$ compared to sustained administration of $10 \mathrm{nM}$ DEX with an average force of $147.7 \pm 31.8 \mu \mathrm{N}$ (49.3\% difference); however, the variability within the group with sustained administration of $10 \mathrm{nM}$ DEX reduced the ability to identify whether a significant difference between the 2 groups was present. Similarly, there were no differences in force production between the $25 \mathrm{nM}$ DEX-receiving groups. In addition, the contractile properties, TPT and $1 / 2 \mathrm{RT}$, of the SMU were measured and no differences were found between the groups (Table 1). Together, the most structurally and functionally mature SMU were obtained with sustained administration of $10 \mathrm{nM} \mathrm{DEX} \mathrm{(days} \mathrm{0-16).}$

\section{Discussion}

The purpose of this study was to investigate the myogenic potential of $25 \mathrm{nM}$ DEX by examining the effects in vitro following DEX treatment at early proliferation (days $0-4$ ), late proliferation (days 3-5), and early differentiation (days 5-7) stages of myogenesis. We hypothesized that administration of $25 \mathrm{nM}$ DEX during an optimal time point of our fabrication process would yield the greatest advancement in structural and functional properties of our engineered SMU, exceeding previously evaluated improvements resulting from the administration of sustained 10 nM DEX.

To fabricate SMU that mimic the phenotype of adult muscle, the early stages of development must be carefully mediated to induce satellite cell proliferation, myoblast differentiation, and myotube fusion. Examination of the DEX dose on early proliferation (days 0-4) showed significantly more myogenic cell proliferation with $25 \mathrm{nM}$ DEX compared to $10 \mathrm{nM}$ DEX. In addition, myoblast differentiation was enhanced with administration of $25 \mathrm{nM}$ DEX at early differentiation (days 5-7) and sustained administration of $10 \mathrm{nM}$ DEX (days 0-7) compared to administration of $25 \mathrm{nM}$ DEX at early and late stages of proliferation. The improvement in myoblast differentiation, with administration of $25 \mathrm{nM}$ DEX at early differentiation and sustained administration of $10 \mathrm{nM} \mathrm{DEX}$, translated to the dense monolayers containing myotubes with the greatest fusion indexes and diameters. In addition, visu- 
Table 1. Comparison of contractile properties of DEX-supplemented SMU

\begin{tabular}{lll}
\hline & TPT, ms & $1 / 2$ RT, ms \\
\hline Sustained 10 nM DEX on days 0-16 & $476.3 \pm 37.0$ & $334.7 \pm 19.5$ \\
Early proliferation 25 nM DEX on days 0-4 & $584.0 \pm 93.0$ & $397.0 \pm 39.8$ \\
Late proliferation 25 nM DEX on days 3-5 & $489.3 \pm 68.9$ & $337.0 \pm 16.6$ \\
Early differentiation 25 nM DEX on days 5-7 & $545.3 \pm 11.3$ & $334.7 \pm 19.5$ \\
\hline
\end{tabular}

Values are means \pm SEM. alization of monolayers and electron micrographs of SMU revealed the greatest advancement in sarcomeric structure with sustained administration of $10 \mathrm{nM}$ DEX; however, electron micrographs of SMU receiving $25 \mathrm{nM}$ DEX at early differentiation showed increased rough endoplasmic reticulum in the cytoplasm of myocytes and increased nucleoli in myonuclei, indicating protein synthesis. Furthermore, the TEM images of SMU administered $25 \mathrm{nM}$ DEX at early differentiation suggested predominantly myogenic growth and organization rather than fibrogenic growth due to the abundance of myofibers and decrease in ECM. Ultimately, the increase in protein synthesis suggests that the SMU administered 25 nM DEX at early differentiation were beginning to develop more myofibers and sarcomeric organization, which may produce more advanced structural properties than SMUs with sustained administration of $10 \mathrm{nM}$ DEX if provided more time to develop. Overall, sustained administration of $10 \mathrm{nM}$ DEX (days 0-16) improved myogenic differentiation and structural maturation, yielding SMU with the greatest force production.

Evaluation of the contractile properties of our SMU showed that isometric force production was enhanced with administration of $25 \mathrm{nM} \mathrm{DEX}$ at early differentiation (days 5-7) and sustained administration of $10 \mathrm{nM}$ DEX (days 0-16); however, no differences were found between the DEX-receiving groups with TPT and $1 / 2$ RT. Consequently, our data suggests that the timing and dose of DEX supplemented to our SMU do not play a significant role in the calcium dynamics. However, it has been shown that changes in serum origin, glucose concentration, and electrical stimulation alter key determinants of TPT and $1 / 2 \mathrm{RT}$, such as myosin heavy chain, troponin, and SERCA isoforms [Dennis et al., 2001; Huang et al., 2006; Khodabukas and Baar, 2014, 2015]. Based on our previous study showing enhanced function with DEX addition, these findings of altered time-dependent contractile properties with growth factors suggest that further investigation should be done to determine whether or not the administration of DEX effects these parameters. Ultimately, in our DEX-receiving SMU, it would be beneficial to measure the expression levels of proteins responsible for calcium release and reuptake in muscle to determine if DEX administration to our SMU changes the expression of these proteins that correlate to calcium dynamics. Subsequently, whether these changes at the protein level have functional consequences could be confirmed.

The administration of sustained $10 \mathrm{nM}$ DEX advanced myogenesis and structural maturation, which supports the results observed in our previous evaluation of DEX. However, in comparison to our previous results, sustained administration of $10 \mathrm{nM}$ DEX produced an average isometric tetanic force $73.7 \%$ greater than the average isometric tetanic force produced with sustained administration of $10 \mathrm{nM}$ DEX in this study $(256.5$ vs. $147.7 \mu \mathrm{N}$, respectively) [Syverud et al., 2016]. It is important to note that in this study sustained administration of $10 \mathrm{nM}$ DEX exhibited a large variation in the forces measured in constructs from different cell isolations; comparison of forces between isolations showed a range of tetanic forces from 50.7 to $270.5 \mu \mathrm{N}$. Potential explanations for the considerable variation in tetanic force are the differences in purity of satellite cell population at the time of seeding. Therefore, we are currently developing techniques to increase the purity of the percentage of satellite cells in our starting cell source to subsequently reduce variability in construct fabrication times and outcomes such as force production. In particular, it is important to force test the constructs when they are composed of mature and aligned myofibers, since contractile function of the construct is determined by the muscle architecture.

Furthermore, with sustained administration of $25 \mathrm{nM}$ DEX the cells exhibited extensive membrane blebbing, a biomarker for apoptosis [Ward et al., 2008; Syverud et al., 2016]. While sustained administration of $25 \mathrm{~nm}$ DEX appeared to produce a toxic environment, single-dose administration of $25 \mathrm{nM}$ DEX during a precise stage of myogenesis reduced the amount of blebbing, yet it is insufficient to produce optimal SMU with respect to structural maturity and functionality. Further analysis is necessary 
to assess the source of blebbing and to determine if administration of $25 \mathrm{nM} \mathrm{DEX}$ at more than one time point of our fabrication process could produce SMU with a more advanced sarcomeric structure and a higher force production. Specifically, SMU with the most advanced structure and function may be produced from administration of $10 \mathrm{nM}$ DEX continuously with administration of $25 \mathrm{nM}$ DEX during early differentiation (days 5-7) or administration of $10 \mathrm{nM}$ DEX continuously with administration of $25 \mathrm{nM} \mathrm{DEX}$ during the majority of early proliferation (days 0-3) and early differentiation (days 5-7). Overall, these observations reinforce the importance of developing a strict methodology for engineering skeletal muscle.

In conclusion, we found that altering the timing of 25 nM DEX administration did not enhance the overall structure or function of our SMU, and SMU were optimally fabricated with sustained administration of $10 \mathrm{nM}$ DEX. Overall, supplementation of DEX to our muscle isolates in vitro has improved our skeletal muscle fabrication protocol by enhancing the myogenesis, structural maturation, and force production of our SMU developing more adult-like muscle. Future studies will utilize $10 \mathrm{nM}$ DEX and examine the effects of other growth factors with myogenic potential in vitro.

\section{Acknowledgements}

The authors would like to acknowledge contributions by Alejandro Moncada, as well as the support of the NIH/NIAMS (1R01AR067744-01), internal funding from the Rackham Graduate School, University of Michigan, and the University of Michigan Microscopy, Imaging and Analysis Laboratory (MIL).

\section{Disclosure Statement}

The authors have no competing financial interests.

\section{References}

Allen, R.E., L.K. Boxhorn (1989) Regulation of skeletal muscle satellite cell proliferation and differentiation by transforming growth factor-beta, insulin-like growth factor I, and fibroblast growth factor. J Cell Physiol 138: 311-315.

Alway, S.E., S.L. Pereira, N.K. Edens, Y. Hao, B.T. Bennett (2013) $\beta$-Hydroxy- $\beta$-methylbutyrate (HMB) enhances the proliferation of satellite cells in fast muscle of aged rats during recovery from disuse atrophy. Exp Geront 48: 973984.

Belanto, J.J., S.V. Diaz-Perez, C.E. Magyar, M.M. Maxwell, Y. Yilmaz, K. Topp, G. Boso, C.H. Jamieson, N.A. Cacalano, C.A.M. Jamieson (2010) Dexamethasone induces dysferlin in myoblasts and enhances their myogenic differentiation. Neuromuscul Disord 20: 111121.

Dennis, R.G., P.E. Kosnik (2000) Excitability and isometric contractile properties of mammalian skeletal muscle constructs engineered in vitro. In Vitro Cell Dev Biol Anim 36: 327-335.

Dennis, R.G., P.E. Kosnik, M.E. Gilbert, J.A Faulkner (2001) Excitability and contractility of skeletal muscle engineered from primary cultures and cell lines. Am J Physiol Cell Physiol 280: 288-295.

Desler, M.M., S.J. Jones, C.W. Smith, T.L. Woods (1996) Effects of dexamethasone and anabolic agents on proliferation and protein synthesis and degradation in C2C12 myogenic cells. J Anim Sci 74: 1265-1273.

Giorgino, F., R.J. Smith (1995) Dexamethasone enhances insulin-like growth factor-1 effects on skeletal muscle cell proliferation. J Clin Invest 96: 1473-1483.
Huang, Y.C., Dennis, R.G., K. Baar (2006) Cultured slow vs. fast skeletal muscle cells differ in physiology and responsiveness to stimulation. Am J Physiol Cell Physiol 291: 11-17.

Husmann, I., L. Soulet, J. Gautron, I. Martelly, D. Barritault (1996) Growth factors in skeletal muscle regeneration. Cytokine Growth Factor Rev 7: 249-258.

Inder, W.J., C. Jang, V.R. Obeyesekere, F.P. Alford (2010) Dexamethasone administration inhibits skeletal muscle expression of the androgen receptor and IGF-1 - implications for steroid-induced myopathy. Clin Endocrinol (Oxf) 73: 126-132.

Khodabukas, A., K. Baar (2014) The effect of serum origin on tissue engineered skeletal muscle function. J Cell Biochem 115: 2198-2207.

Khodabukas, A., K. Baar (2015) Contractile and metabolic properties of engineered skeletal muscle derived from slow and fast phenotype mouse muscles. J Cell Physiol 230: 1750-1757.

Kuo, T., P.H. Lui, T. Chen, R.A. Lee, J. New, D. Zhang, C. Lei, A. Chau, Y. Tang, E. Cheung, J. Wang (2016) Transcriptional regulation of $\mathrm{FoxO}_{3}$ gene by glucocorticoids in murine myotubes. Am J Physiol Endocrinol Metab 310: 572-585.

Larkin, L.M., S. Calve, T.Y. Kostrominova, E.M. Arruda (2006) Structure and functional evaluation of tendon-skeletal muscle constructs engineering in vitro. Tissue Eng 12: 3149-3158.

Larkin, L.M., W.M. Kuzon Jr., M.A. Supiano, A. Galecki, J.B. Halter (1998) Effect of age and neurovascular grafting on the mechanical function of medial gastrocnemius muscles of Fischer 344 rats. J Gerontol A Biol Sci Med Sci 53: B252-B258.
Mertens, J.P., K.B. Sugg, J.D. Lee, L.M. Larkin (2014) Engineering muscle constructs for the creation of functional engineered musculoskeletal tissue. Regen Med 9: 89-100.

Morgan, S.A., Z.K. Hassan-Smith, C.L. Doig, M. Sherlock, P.M. Stewart, G.G. Lavery (2016) Glucocorticoids and $11 \beta$-HSD1 are major regulators of intramyocellular protein metabolism. J Endocrinol 229: 277-286.

National Research Council (1996) Guide for the care and use of laboratory animals. Washington, National Academics Press.

Qin, J., R. Du, Y. Yang, H. Zhang, Q. Li, L. Liu, H. Guan, J. Hou, X. An (2013) Dexamethasoneinduced skeletal muscle atrophy was associated with upregulation of myostatin promoter activity. Res Vet Sci 94: 84-89.

Schakman, O., H. Gilson, J.P. Thissen (2008) Mechanisms of glucocorticoid-induced myopathy. J Endocrinol 197: 1-10.

Syverud, B.C., K.W. VanDusen, L.M. Larkin (2016) Effects of dexamethasone on satellite cells and tissue engineered skeletal muscle units. Tissue Eng Part A 22: 480-490.

VanDusen, K.W., B.C. Syverud, M.L. Williams, J.D. Lee, L.M. Larkin (2014) Engineered skeletal muscle units for repair of volumetric muscle loss in the tibialis anterior muscle of a rat. Tissue Eng Part A 20: 2920-2930.

Ward, T.H., J. Cummings, E. Dean, A. Greystroke, J.M. Hou, A. Backen, M. Ranson, C. Dive (2008) Biomarkers of apoptosis. Br J Cancer 99: 841-846.

Williams, M.L., T.Y. Kostrominova, E.M. Arruda, L.M. Larkin (2013) Effects of implantation on engineered skeletal muscle constructs. J Tissue Eng Regen Med 7: 434-442. 